\title{
Scattered-Field FDTD Algorithm for Hot Anisotropic Plasma With Application to EC Heating
}

\author{
Christos Tsironis, Theodoros Samaras, Member, IEEE, and Loukas Vlahos
}

\begin{abstract}
Nowadays, the finite-difference time-domain (FDTD) is accepted as a reliable tool in numerical electromagnetics. In the field of wave propagation in plasmas, the mainstream in theory and applications is oriented to frequency domain asymptotic methods, where the determination of the plasma response presents less difficulty. However, in many cases of interest (like, e.g., mode conversion) this approach breaks down, the solution becomes questionable and therefore a full-wave analysis is necessary. In this work, we present a (novel) scattered-field FDTD algorithm for fully kinetic, anisotropic plasma. As an application, we study the perpendicular electron-cyclotron propagation and absorption in simplified tokamak geometry, under different physics models for the dielectric response of the plasma. In general, since FDTD is a time domain technique, conversion from the frequency domain is needed in order to be able to exploit the existing knowhow on the dielectric response. However, in the case of a constant-frequency wave propagating in a stationary plasma, the FDTD method can be applied directly using the frequency domain dielectric tensor.
\end{abstract}

Index Terms-Anisotropic plasma, electron-cyclotron (EC) heating, finite-difference time-domain (FDTD), hot plasma dispersion, scattered-field.

\section{INTRODUCTION}

$\mathbf{T}$ HE injection of electron-cyclotron (EC) waves is a standard method for coupling energy to plasma electrons in modern fusion devices (tokamaks, stellarators), with primary applications the plasma heating (ECRH) and the noninductive current drive (ECCD) (see [1] and references therein). In fusion experiments, the EC waves are launched in the plasma in the form of spatially narrow beams, and interact with the electrons when the EC resonance condition is fulfilled

$$
\omega-k_{\|} v_{\|}-\frac{l \omega_{c}}{\gamma}=0(l= \pm 1, \pm 2, \ldots)
$$

where $\omega$ is the wave frequency, $\omega_{c}=e B_{0} / m_{e}$ is the cyclotron frequency ( $B_{0}$ is the magnetic field), $k_{\|}, v_{\|}$are the wavenumber and the electron velocity components parallel to the magnetic field, and $\gamma$ the Lorentz factor. Since the cyclotron frequency is proportional to the magnetic field, which is nonuniform in fusion devices, this condition is satisfied in a narrow spatial region called "resonance layer."

Manuscript received July 23, 2007; revised January 1, 2008. Published September 4, 2008 (projected). This work was supported by the EURATOM Association-Hellenic Republic.

The authors are with the Department of Physics, Aristotle University of Thessaloniki, Thessaloniki 54006, Greece (e-mail: ctsironis@ astro.auth.gr).

Digital Object Identifier 10.1109/TAP.2008.928774
The propagation of EC waves in the plasma is described by Maxwell's equations. In general, to obtain a full solution to the problem is burdensome because these equations are partial differential (PDE). In numerical applications, a PDE is equivalent to a spatial grid progressing on a time grid, which for the wave and plasma parameters occurring in fusion may be very resource-demanding, in some cases prohibitively. When the wavelength is small compared to the scale length of inhomogeneity of the plasma, a simplification is reached by frequency-domain asymptotic methods: ray tracing [2], quasi-optics [3] or beam tracing [4]. The solution is obtained over Hamiltonian differential equations, where the dispersion function plays the role of the Hamiltonian. The plasma response is derived in terms of the linear theory of plasma oscillations [5], based on that for typical experimental parameters the wave intensity is small and falls in the linear regime.

In asymptotic methods, dispersion modelling follows the standard approach of calculating wave trajectories using the Appleton-Hartree (cold plasma) dispersion relation [5]. The motivation has been the fact that, to leading order, the trajectories near the cyclotron resonance are exactly those of cold plasma theory. However, in cases of particular interest like the perpendicular O-mode near fundamental resonance or the $\mathrm{X}$-mode near the 2nd-harmonic, the contributions of the higher orders become very large. Recently, both experimental and theoretical evidence is pointing to the possible importance of hot plasma dispersion near resonance [6], [7]. Particularly in [7], the direction of ray propagation is shown to differ dramatically from the cold plasma trajectory. This implies effects on the evolution of the polarization vector, especially near resonance, and thus on the absorption of the wave. In such cases, a full-wave treatment is called for.

The finite-difference time-domain (FDTD) method is nowadays recognized as a reliable tool in numerical electromagnetism. However, for realistic ECRH simulations, the required spatial resolution makes the computational requirements very large, and that is why there has been little application of FDTD to those problems up to day. Among the existing FDTD literature on hot plasma, worthy of mention are the 1D simulations of interferometry in [8] assuming cold plasma propagation but taking into account collisional and non-relativistic cyclotron damping, followed by 3D simulations where the plasma response is described in terms of the electric polarization [9]. Also of interest are the 2D simulations of reflectometry performed in cold plasma with elongated magnetic geometry [10], and the simulations of fundamental ECRH in [11] where the plasma response is described by an "artificial" conductivity tensor based on fluid theory. 
In this work, we present a (novel) scattered-field FDTD algorithm for hot anisotropic plasma. For a constant-frequency wave propagating in stationary plasma, FDTD can be applied directly exploiting knowledge from the frequency domain. A numerical application is presented in plane geometry, allowing for a simpler description of the plasma-wave coupling based on the 1D implementation of FDTD. The structure of the paper is as follows: In Section II we generalize the standard scattered-field FDTD formulation in order to treat anisotropic materials. The propagation and absorption in simplified geometry are studied in Section III, under different implementations of the plasma dielectric tensor (cold, warm, and hot weakly-relativistic) in order to ascertain the importance of the different physics in the modelling. Finally, in Section IV we summarize the conclusions.

\section{SCATTERED-FIELD FDTD FORMALISM FOR ANISOTROPIC} PLASMA

In the Yee formulation [12], Maxwell's equations are discretized and solved for the total fields. Alternatively, in a scattered-field formalism, the total field is assumed as the superposition of an incident and a scattered field, $\mathbf{E}=\mathbf{E}_{\mathbf{i}}+\mathbf{E}_{\mathbf{s}}$. The incident field is the field that would exist in the absence of the medium, thought of as always propagating in free space, while the scattered field is generated by the medium in response to the incident field. The rationale for the separate field approach is that the incident field can be specified (or solved) analytically throughout the problem space. This simplifies the numerical procedure, because only the scattered fields need to be absorbed at the boundaries of the computational domain, which are truncated with Mur's outer-radiating boundary condition (ORBC) [13].

We are interested in applying FDTD, as a wave solver, to the modelling of EC heating. The fusion plasma, as a medium, may be assumed stationary and linear, however it is anisotropic due to the axial magnetic field. Therefore, it is necessary to generalize the Yee formulation in order to treat anisotropic materials. Mathematically, this means the introduction of tensor, instead of scalar, plasma response. The total-field formalism for anisotropic medium has been derived by Schneider and Hudson [14]. In their paper, FDTD is extended to accommodate nonzero off-diagonal elements in the permittivity and conductivity, and the resulting equations differ substantially from the ones of Yee. Following this spirit, we present the scattered-field formulation for the problem.

In anisotropic plasma, the dielectric displacement is related to the electric field by a permittivity tensor and the current density by a conductivity tensor

$$
\mathbf{D}=\overline{\bar{\varepsilon}} \mathbf{E}, \mathbf{j}=\overline{\bar{\sigma}} \mathbf{E}
$$

Combining the above equations with Maxwell's equations, one finds the curl equations for the total fields, from which it is straightforward to obtain the equations for the scattered fields

$$
\begin{aligned}
\nabla \times \mathbf{E}_{\mathbf{s}} & =-\mu_{0} \frac{\partial \mathbf{H}_{\mathbf{s}}}{\partial t} \\
\nabla \times \mathbf{H}_{\mathbf{s}} & =\overline{\bar{\sigma}} \mathbf{E}_{\mathbf{s}}+\overline{\bar{\varepsilon}} \frac{\partial \mathbf{E}_{\mathbf{s}}}{\partial t}+\overline{\bar{\sigma}} \mathbf{E}_{\mathbf{i}}+\left(\overline{\bar{\varepsilon}}-\varepsilon_{0} \overline{\bar{I}}\right) \frac{\partial \mathbf{E}_{\mathbf{i}}}{\partial t} .
\end{aligned}
$$

The discretization of (3) follows, based on the leap-frog scheme. For simplicity, we adopt the use of cubic cells $(\Delta x=\Delta y=\Delta z=\Delta r$ ), however the results are easily generalized to nonuniform grid geometries. By defining the following "curl" vector-matrix (sum on the values $m=-1,0,1$ )

$$
\mathbf{\Psi}\left[\left.\mathbf{A}\right|_{i, j, k} ^{n}\right]=\sum_{m=-1}^{1} m\left[\begin{array}{l}
\left.A_{z}\right|_{i, j+m / 2, k} ^{n}-\left.A_{y}\right|_{i, j, k+m / 2} ^{n} \\
\left.A_{x}\right|_{i, j, k+m / 2} ^{n}-\left.A_{z}\right|_{i+m / 2, j, k} ^{n} \\
\left.A_{y}\right|_{i+m / 2, j, k} ^{n}-\left.A_{x}\right|_{i, j+m / 2, k} ^{n}
\end{array}\right]
$$

the discrete equations of (3a) in each direction $(q=x, y, z)$ may be written in compact form as

$$
\left.H_{q s}\right|_{i, j, k} ^{n+1 / 2}=\left.H_{q s}\right|_{i, j, k} ^{n-1 / 2}-\frac{\Delta t}{\mu_{0} \Delta r} \Psi_{q}\left[\left.\mathbf{E}_{\mathbf{s}}\right|_{i, j, k} ^{n}\right] .
$$

For (3b) things are not so simple. The electric field cannot be updated directly, as was done for the magnetic field, because the off-diagonal terms of $\overline{\bar{\varepsilon}}, \overline{\bar{\sigma}}$ cause the temporal derivatives of the electric field to appear on the right-hand side, and therefore the updating equations are coupled. To formulate the time-stepping of $\mathbf{E}_{\mathbf{s}}$, these equations must be solved as an algebraic system. The derivation of the exact expressions is rather cumbersome, however it is a matter of straightforward calculus. We define the tensors

$$
\overline{\bar{s}}=\frac{\overline{\bar{\varepsilon}}}{\Delta t}-\frac{\overline{\bar{\sigma}}}{2}, \overline{\bar{a}}=\left(\frac{\overline{\bar{\varepsilon}}}{\Delta t}+\frac{\overline{\bar{\sigma}}}{2}\right)^{-1} .
$$

This simplifies the expressions for the components of $\left.\mathbf{E}_{\mathbf{s}}\right|_{i, j, k} ^{n+1}$ to a compact form such like (5)

$$
\begin{aligned}
\left.E_{q s}\right|_{i, j, k} ^{n+1}= & \left.\sum_{l=1}^{3} \sum_{m=1}^{3} a_{q l}\right|_{i, j, k}\left\{\left.\left.s_{l m}\right|_{i, j, k} E_{m s}\right|_{i, j, k} ^{n}\right. \\
& +\Psi_{q}\left[\left.\mathbf{H}_{\mathbf{s}}\right|_{i, j, k} ^{n+1 / 2}\right]-\left.\left.\sigma_{l m}\right|_{i, j, k} E_{m i}\right|_{i, j, k} ^{n+1 / 2} \\
& \left.-\left.\left(\left.\varepsilon_{l m}\right|_{i, j, k}-\varepsilon_{0} \delta_{l m}\right) \frac{\partial E_{m i}}{\partial t}\right|_{i, j, k} ^{n+1 / 2}\right\} .
\end{aligned}
$$

With the derivation of (5) and (7), our job on the discretization is not completely done. Unlike the Yee equations, in (7) the fields on the right-hand side are not "automatically" available at the positions demanded, due to the appearance not just of the electric field component along the axis considered but also of the other two components. That is induced by the anisotropy of the medium and cannot be automatically resolved by the Yee algorithm (e.g., by changing the structure of the grid). For example, the computation of $\left.E_{z s}\right|_{i, j, k} ^{n+1}$ requires $\left.E_{x s}\right|_{i, j, k} ^{n},\left.E_{y s}\right|_{i, j, k} ^{n}$ and $\left.H_{x s}\right|_{i, j, k \pm 1 / 2} ^{n+1 / 2},\left.H_{y s}\right|_{i, j, k \pm 1 / 2} ^{n+1 / 2},\left.H_{z s}\right|_{i \pm 1 / 2, j, k} ^{n+1 / 2},\left.H_{z s}\right|_{i, j \pm 1 / 2, k} ^{n+1 / 2}$, however these values are not available directly from the FDTD grid. The electric field quantities $E_{q s}(q=x, y, z)$ are found at the locations $\left(i+\delta_{q x} / 2, j+\delta_{q y} / 2, k+\delta_{q z} / 2\right)$, while the magnetic field at $\left(i+\delta_{q x}^{\prime} / 2, j+\delta_{q y}^{\prime} / 2, k+\delta_{q z}^{\prime} / 2\right)\left(\delta_{i j}^{\prime}=1-\delta_{i j}\right.$ is the complement of Kronecker's delta). To calculate the needed values for $\left.E_{z s}\right|_{i, j, k} ^{n+1}$, one adopts interpolating approximations in 
space among the locations of interest. As a demonstration, we give the result for the values of $\left.E_{x s}\right|_{i, j, k} ^{n}$

$$
\begin{aligned}
\left.E_{x s}\right|_{i, j, k} ^{n}= & \frac{1}{4}\left(\left.E_{x s}\right|_{i+1 / 2, j, k+1 / 2} ^{n}+\left.E_{x s}\right|_{i+1 / 2, j, k-1 / 2} ^{n}\right. \\
& \left.+\left.E_{x s}\right|_{i-1 / 2, j, k+1 / 2} ^{n}+\left.E_{x s}\right|_{i-1 / 2, j, k-1 / 2} ^{n}\right) .
\end{aligned}
$$

To complete the treatment, one needs to specify the plasma response tensors. The plasma reaction to EC waves is stimulated by the physics of electron motions. Therefore, in principle, a kinetic treatment is called for. It is beyond the scope of this work to go into the details of the calculations; for the full derivation and explanations the reader is referred to [5]. Here, the main steps are just sketched: (a) linearization of the kinetic (Vlasov) equation assuming a homogeneous equilibrium plus a small-amplitude Fourier perturbation; (b) solution for the distribution function by integrating along the unperturbed orbits in velocity space; (c) calculation of the current density as the 1st-order moment of the distribution; and (d) determination of the response tensors in Fourier space by fitting the result for the current density to Ohm's law.

It is customary to express the plasma response to electromagnetic waves in terms of a complex dielectric tensor, which is related to the real permittivity and conductivity tensors introduced in (2) as follows:

$$
\varepsilon_{0} \overline{\bar{\epsilon}}=\overline{\bar{\varepsilon}}+i \frac{\overline{\bar{\sigma}}}{\omega}
$$

or, inversely, $\overline{\bar{\varepsilon}}=\varepsilon_{0} \operatorname{Re}(\overline{\bar{\epsilon}})$ and $\overline{\bar{\sigma}}=\omega \varepsilon_{0} \operatorname{Im}(\overline{\bar{\epsilon}})$. The dimensionless tensor is of the form

$$
\begin{aligned}
\overline{\bar{\epsilon}}=\overline{\bar{I}}+\frac{\omega_{p}^{2}}{\omega} \sum_{l=-\infty}^{\infty} \int d^{3} p \frac{\frac{1}{\gamma p} \frac{d f_{0}}{d p}}{\omega-k_{\|} v_{\|}-\frac{l \omega_{c}}{\gamma}} \\
\cdot\left[\begin{array}{ccc}
\frac{l^{2}}{b^{2}} J_{l}^{2} p_{\perp}^{2} & i \frac{l}{b} J_{l} J_{l}^{\prime} p_{\perp}^{2} & \frac{l}{b} J_{l}^{2} p_{\|} p_{\perp} \\
-i \frac{l}{b} J_{l} J_{l}^{\prime} p_{\perp}^{2} & \left(J_{l}^{\prime}\right)^{2} p_{\perp}^{2} & -i J_{l} J_{l}^{\prime} p_{\|} p_{\perp} \\
\frac{l}{b} J_{l}^{2} p_{\|} p_{\perp} & i J_{l} J_{l}^{\prime} p_{\|} p_{\perp} & J_{l}^{2} p_{\|}^{2}
\end{array}\right] .
\end{aligned}
$$

In the above, $\omega_{p}^{2}=n_{e} e^{2} /\left(\varepsilon_{0} m_{e}\right)$ is the plasma frequency $\left(n_{e}\right.$ the electron density), $f_{0}(p)$ is the equilibrium distribution function $\left(p^{2}=p_{\|}^{2}+p_{\perp}^{2}\right)$ and $J_{l}(b)$ the Bessel function of order $l$ and argument $b=k_{\perp} p_{\perp} /\left(m_{e} \omega_{c}\right)$. Notice the symmetry (Onsager) relations $\epsilon_{x y}=-\epsilon_{y x}, \epsilon_{x z}=\epsilon_{z x}$ and $\epsilon_{y z}=-\epsilon_{z y}$. The momentum space integrals are calculated according to the Landau prescription, i.e., over the poles caused by the zeros of the denominators. In order to obtain the exact expression of the dielectric tensor one has to insert in (10) the Maxwellian distribution function in the place of $f_{0}$ and perform the integrations. For the fully-relativistic case, the evaluation of the dielectric tensor requires numerical effort. To obtain analytic, more tractable expressions of the dielectric tensor, one should resort to certain approximations.

The non-relativistic approximation amounts to setting $\gamma=1$ and using the classical-physics version of the Maxwellian. In this case, the resonant denominator is independent of $p_{\perp}$ and the integration over $p_{\|}$is carried out analytically. The validity of this approach requires that $\left|N_{\|}\right| \gg v_{t h} / c,\left|1-l \omega_{c} / \omega\right|$
$\left(\mathbf{N}=c \mathbf{k} / \omega\right.$ is the refraction index and $v_{t h}^{2}=k_{B} T_{e} / m_{e}$ the thermal velocity), which guarantee that resonant electrons remain sub-relativistic and that the Doppler effect dominates the relativistic frequency downshift. When these conditions break down, as e.g., in the case of perpendicular propagation $\left(N_{\|}=0\right)$, one should upgrade to the weakly-relativistic approach, where a Taylor expansion in the momentum is employed in the Lorentz factor. The velocity space integration yields for the dielectric tensor elements

$$
\begin{aligned}
\epsilon_{x x}= & 1-\mu \frac{\omega_{p}^{2}}{\omega^{2}} \sum_{l=-\infty}^{\infty} \frac{l^{2}}{\lambda} \Gamma_{|l|} \mathcal{F}_{|l|+3 / 2} \\
\epsilon_{x y}= & -i \mu \frac{\omega_{p}^{2}}{\omega^{2}} \sum_{l=-\infty}^{\infty} l \Gamma_{|l|}^{\prime} \mathcal{F}_{|l|+3 / 2} \\
\epsilon_{x z}= & \mu \frac{\omega_{p}^{2}}{\omega \omega_{c}} N_{||} N_{\perp} \sum_{l=-\infty}^{\infty} \frac{l}{\lambda} \Gamma_{|l|}\left(\mathcal{F}_{|l|+3 / 2}-\mathcal{F}_{|l|+5 / 2}\right) \\
\epsilon_{y y}= & 1-\mu \frac{\omega_{p}^{2}}{\omega^{2}} \sum_{l=-\infty}^{\infty}\left(\frac{l^{2}}{\lambda} \Gamma_{|l|} \mathcal{F}_{|l|+3 / 2}+2 \lambda \Gamma_{|l|}^{\prime} \mathcal{F}_{|l|+5 / 2}\right) \\
\epsilon_{y z}= & i \mu \frac{\omega_{p}^{2}}{\omega \omega_{c}} N_{\|} N_{\perp} \sum_{l=-\infty}^{\infty} \Gamma_{|l|}^{\prime} \mathcal{F}_{|l|+5 / 2} \\
\epsilon_{z z}= & 1-\mu \frac{\omega_{p}^{2}}{\omega^{2}} \sum_{l=-\infty}^{\infty} \Gamma_{|l|}\left[\mu N _ { | | } ^ { 2 } \left(\mathcal{F}_{|l|+7 / 2}\right.\right. \\
& \left.\left.-2 \mathcal{F}_{|l|+5 / 2}+\mathcal{F}_{|l|+3 / 2}\right)+\mathcal{F}_{|l|+5 / 2}\right]
\end{aligned}
$$

In the above, $\mu=c^{2} /\left(2 v_{\mathrm{th}}^{2}\right)$ is the inverse of the normalized thermal energy, $\Gamma_{l}(\lambda)=e^{-\lambda} I_{l}(\lambda)$ with $I_{l}(\lambda)$ the modified Bessel function of argument $\lambda=k_{\perp}^{2} v_{\mathrm{th}}^{2} / \omega_{c}^{2}$, and $\mathcal{F}_{q}\left(\alpha, \zeta_{l}\right)$ are the Shkarofsky functions of arguments $\alpha=\mu N_{\|}^{2} / 2$ and $\zeta_{l}=\mu\left(1-l \omega_{c} / \omega\right)[15]$.

At this point it is necessary to comment on some aspects. First, from (11) one sees that a finite temperature introduces a dependence of the dielectric tensor on the refraction index. Therefore, apart from the case of cold plasma, the refraction index is necessary for calculating the tensor elements. The calculation of $\mathbf{N}$ is done from the dispersion relation, which in general form reads

$$
\left|-N^{2} \overline{\bar{I}}+\mathbf{N N}+\overline{\bar{\varepsilon}}\right|=0
$$

where $\mathbf{N N}$ is a dual tensor based on $\mathbf{N}$. The dispersion relation emerges as the solvability condition for the wave equation, which results from Maxwell's equations when one attempts to derive separate relations for $\mathbf{E}$ and $\mathbf{B}$. In general, the dispersion relation appears several branches of solutions. For a certain problem, one has to keep the solution corresponding to the specific mode of propagation.

The parameter $\lambda$ measures the ratio of the gyroradius over the wavelength, hence the importance of finite Larmor radius (FLR) effects. By that, we mean effects raised by the inclusion of the cyclotron motions in the description of the plasma response to external fields. In the limit of very small Larmor radius, only the simplest (zero-order) thermal correction is retained, equivalent to a finite pressure term, and both approximations of the 
fully-relativistic tensor reach a form known as the warm plasma dielectric tensor [5]

$$
\begin{aligned}
& \epsilon_{x x}=1-\frac{\omega_{p}^{2}\left(\omega^{2}-k_{\|}^{2} v_{\mathrm{th}}^{2}\right)}{\omega^{2}\left(\omega^{2}-k^{2} v_{\mathrm{th}}^{2}\right)-\omega_{c}^{2}\left(\omega^{2}-k_{\|}^{2} v_{\mathrm{th}}^{2}\right)} \\
& \epsilon_{x y}=i \frac{\omega_{p}^{2} \omega_{c}\left(\omega^{2}-k_{\|}^{2} v_{\mathrm{th}}^{2}\right)}{\omega\left[\omega^{2}\left(\omega^{2}-k^{2} v_{\mathrm{th}}^{2}\right)-\omega_{c}^{2}\left(\omega^{2}-k_{\|}^{2} v_{\mathrm{th}}^{2}\right)\right]} \\
& \epsilon_{x z}=-\frac{\omega_{p}^{2} k_{\|} k_{\perp} v_{\mathrm{th}}^{2}}{\omega^{2}\left(\omega^{2}-k^{2} v_{\mathrm{th}}^{2}\right)-\omega_{c}^{2}\left(\omega^{2}-k_{\|}^{2} v_{\mathrm{th}}^{2}\right)} \\
& \epsilon_{y y}=1-\frac{\omega_{p}^{2}\left(\omega^{2}-k^{2} v_{\mathrm{th}}^{2}\right)}{\omega^{2}\left(\omega^{2}-k^{2} v_{\mathrm{th}}^{2}\right)-\omega_{c}^{2}\left(\omega^{2}-k_{\|}^{2} v_{\mathrm{th}}^{2}\right)} \\
& \epsilon_{z z}=-i \frac{\omega_{p}^{2} \omega_{c} k_{\|} k_{\perp} v_{\mathrm{th}}^{2}}{\omega\left[\omega^{2}\left(\omega^{2}-k^{2} v_{\mathrm{th}}^{2}\right)-\omega_{c}^{2}\left(\omega^{2}-k_{\|}^{2} v_{\mathrm{th}}^{2}\right)\right]}
\end{aligned}
$$

whereas in the limit of zero Larmor radius (no thermal motions) $\overline{\bar{\varepsilon}}$ reduces to the well-known cold plasma form, which may be obtained from (13) by setting $v_{\text {th }}=0$.

\section{NUMERICAL APPLICATION}

We present a numerical application in the perpendicular propagation of an EC beam in simplified tokamak geometry (slab). The simplicity lies in adopting $r_{p} \ll r_{t}$, where $r_{t}, r_{p}$ are the toroidal and poloidal radii. This assumption, known as the large aspect ratio approximation, corresponds to a flat (rather than curved) magnetic geometry with negligent poloidal field. As a consequence, the plasma properties (magnetic field, density and temperature) may be assumed to vary only along the $x$-axis, in the region $\left[-r_{p}, r_{p}\right]$, and the external magnetic field to lie just along $z$. The variation profiles of $\omega_{c}, \omega_{p}$ and $T_{e}$ are of the form

$$
\begin{aligned}
& \omega_{c}(x)=\frac{\omega_{c}(0)}{1+\frac{x}{r_{t}}} \\
& \omega_{p}^{2}(x)=\omega_{p}^{2}(0)+\left[\omega_{p}^{2}\left(r_{p}\right)-\omega_{p}^{2}(0)\right]\left(\frac{x}{r_{p}}\right)^{2} \\
& T_{e}^{2}(x)=T_{e}^{2}(0)+\left[T_{e}^{2}\left(r_{p}\right)-T_{e}^{2}(0)\right]\left(\frac{x}{r_{p}}\right)^{2} .
\end{aligned}
$$

These relations imply that the magnetic field increases from the one plasma edge $x=r_{p}$ (low-field side) to the other $x=-r_{p}$ (high-field side), whereas the density and temperature rise from the edges to the center of the plasma $x=0$.

The incident field is a sinusoidal wave, launched from the low-field side in the negative $x$-direction $\left(k_{x 0}<0, k_{y 0}=\right.$ $\left.k_{z 0}=0\right)$. Since the wave starts in the direction along which the plasma is inhomogeneous, the components of the wavenumber across $x$ remain constantly zero and the propagation stays perpendicular. Regarding FDTD, this allows a simpler (and numerically faster) description of the wave based on the $1 \mathrm{D}$ implementation of the algorithm, obtained by setting $1 / \Delta y=1 / \Delta z=0$ in (5) and (7). The numerical treatment sums up in the solution of the discrete FDTD equations (5), (7) for the 1D case, with dielectric response calculated from (9) according to the specific model, i.e., cold [(13) with $v_{\text {th }}=0$ ], warm [(13)] or hot weakly-relativistic [(11)].

All these are implemented in the code ECFW, which provides the temporal and spatial evolution of the electromagnetic field and the current density in the plasma. For the wave and plasma parameters, we consider the experiments of ASDEX upgrade (AUG) and the International Thermonuclear Experimental Reactor (ITER). In AUG, the plasma radii are $r_{t}=1.65$ $\mathrm{m}, r_{p}=0.6 \mathrm{~m}$, the magnetic field on the axis is $B_{0}(0)=2.5 \mathrm{~T}$, the density at the plasma edge and center is $n_{e}\left( \pm r_{p}\right)=1.4 \cdot 10^{13}$ $\mathrm{cm}^{-3}$ and $n_{e}(0)=1.6 \cdot 10^{13} \mathrm{~cm}^{-3}$, and the analogous values for the temperature are $T_{e}\left( \pm r_{p}\right)=0.2 \mathrm{KeV}$ and $T_{e}(0)=2 \mathrm{KeV}$. The wave frequency is $\omega / 2 \pi=140 \mathrm{GHz}$, corresponding to the 2nd harmonic X-mode, the wave power at injection is $P_{0}=1$ MW and the beam width $w=2 \mathrm{~cm}$. In ITER, the same parameters have values $r_{t}=6.2 \mathrm{~m}, r_{p}=1.9 \mathrm{~m}, B_{0}(0)=5.51$ $\mathrm{T}, n_{e}\left( \pm r_{p}\right)=10^{13} \mathrm{~cm}^{-3}, n_{e}(0)=10^{14} \mathrm{~cm}^{-3}, T_{e}\left( \pm r_{p}\right)=$ $1 \mathrm{KeV}, T_{e}(0)=10 \mathrm{KeV}, \omega / 2 \pi=160 \mathrm{GHz}$ (1st harmonic O-mode), $P_{0}=10 \mathrm{MW}$ and $w=3 \mathrm{~cm}$.

Before presenting the results, we briefly refer to issues on the numerical implementation of FDTD. In the numerical calculation, we follow the procedure listed below.

1) Calculation of the refraction index $\mathbf{N}$ :

a) Replace in (12) the dielectric tensor elements, as given from the model describing the plasma response (cold, warm, hot);

b) Solve the algebraic equation with respect to $\mathbf{N}$;

c) Keep the solution referring to the specific mode of propagation;

2) Calculate the elements of the permittivity and conductivity tensors $\overline{\bar{\varepsilon}}, \overline{\bar{\sigma}}$;

3) Calculate the elements of the tensors $\overline{\bar{s}}, \overline{\bar{a}}$, based on (6);

4) Use these values in order to calculate the electric field through the update equations.

The grid size is taken equal to $1 \%$ of the wavelength in vacuum, whereas the time step is set to $10 \%$ of the stepsize emerging from the Courant stability condition. The stepsizes were given relatively low values with respect to common practice (see, e.g., [16]) because the conductivity in the plasma attains large values, especially near resonance, and therefore the accuracy and stability in the calculations fail for stepsizes relative to usual media. The total integration time is a multiple of the time needed by the wave to cover the poloidal diameter. Since the wave frequency and the dielectric tensor do not depend on time, the calculated fields are expected to reach a steady state. Whether the specified integration time is large enough so that the complete picture is included in the results, is checked by following the temporal evolution of the fields at specific grid points.

In Fig. 1 we show FDTD simulations of cold plasma propagation in AUG. The temporal evolution of the electric field component $E_{x}$ at the checkpoint $x=-0.3 r_{p}$ is plotted in Fig. 1(a). The field is zero up to $\approx 1.2 \cdot 10^{-9} \mathrm{~s}$, the time needed for the wave to arrive at the checkpoint, and indeed reaches a steady-state after $\approx 3.5 \cdot 10^{-9} \mathrm{~s}$ (which correspond to a few more than 3000 wave periods). Since $E_{x}$ is the longitudinal component generated by the plasma, it attains values more than 10 times smaller 


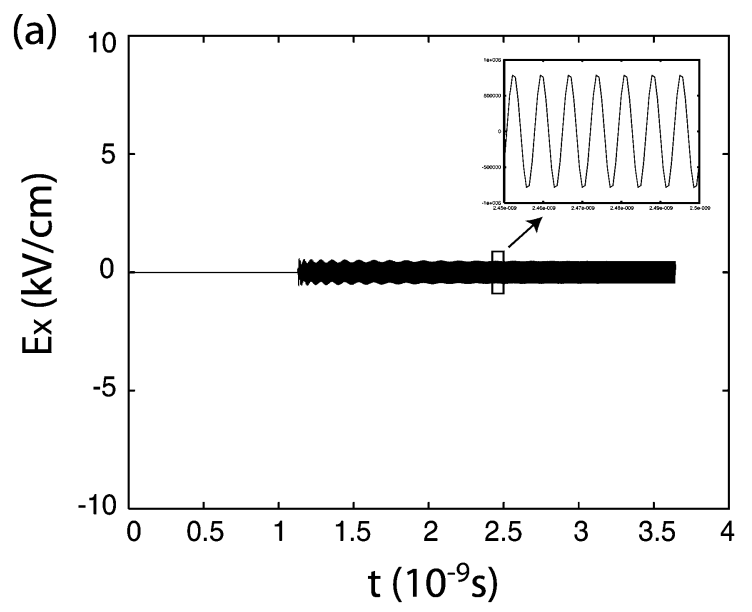

(b)

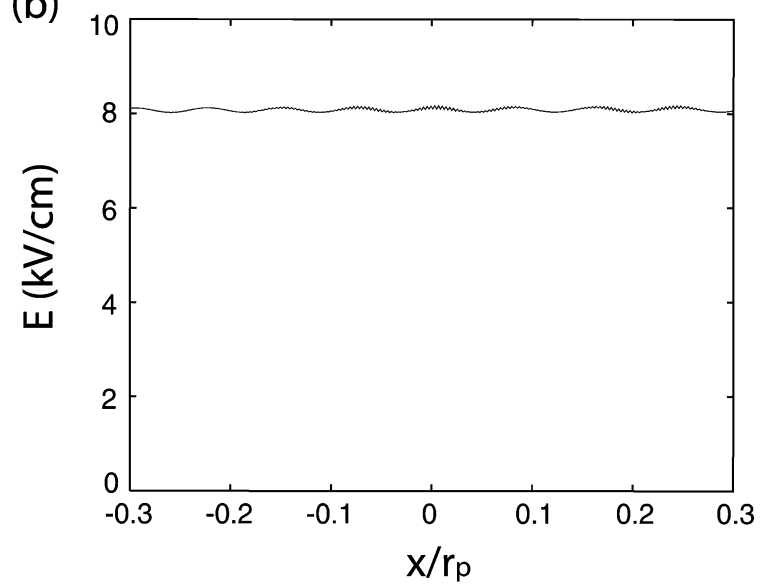

Fig. 1. FDTD simulation of cold plasma for AUG parameters: (a) Time evolution of $E_{x}$ (with a zoom near $t=2.5 \cdot 10^{-9} \mathrm{~s}$ ) at $x=-0.3 r_{p}$, (b) steady-state spatial profile of $E$.

from the amplitude. The fields vary periodically in time, which is not clearly distinguishable before a magnification in Fig. 1(a) (top-right subfigure). In Fig. 1(b) we give the steady-state profile of variation (with respect to $x$ ) of the electric field amplitude $E$. The electric field remains constant along the poloidal, so there is no wave damping in the plasma. This is actually what "cold plasma" means: in the absence of collisions and thermal motions from the modelling, there is no mechanism to absorb the wave energy.

For propagation under the warm plasma model for AUG parameters, in Fig. 2 the spatial profile of $E$ is compared to the same profile in cold plasma [Fig. 1(b)]. It is clear that the plasma response under the cold and warm plasma models is almost identical. This is because the only difference between the cold and warm plasma dielectric tensors is a finite pressure term coming from the lowest-order thermal correction, which however does not describe the collisionless damping owed to resonant electrons. Considering again the case of cold plasma, without collisions into play both the cold and warm plasma tensors are Hermitian and, consequently, contain no wave dissipation. For modelling collisionless absorption, the detailed treatment provided by kinetic theory is necessary.

We studied the case of hot, weakly-relativistic plasma for both AUG and ITER, and the results are presented in Figs. 3 and 4.

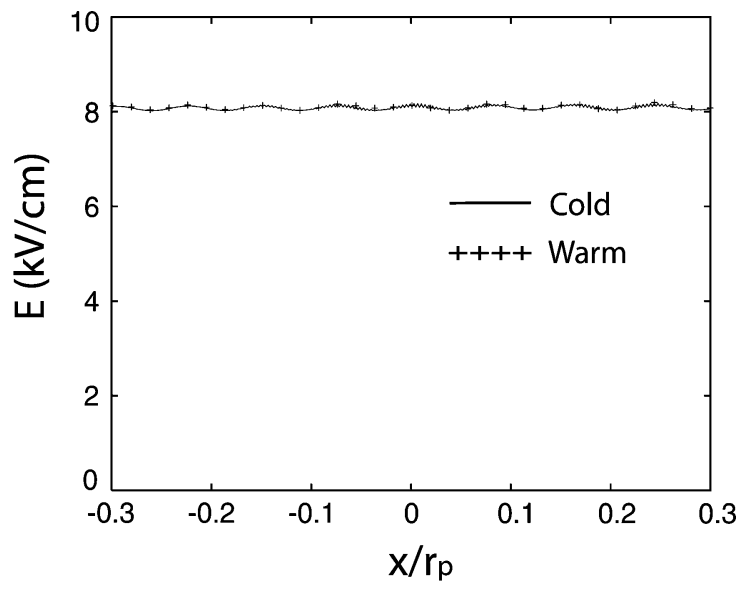

Fig. 2. FDTD simulation of warm plasma for AUG parameters: Steady-state spatial profile of $E$ (in comparison with the cold-plasma profile of Fig. 1).

Whereas the present case is directly connected with ECRH, also the above cases are indirectly related since many algorithms model ECRH as the combination of cold plasma propagation with a hot plasma absorption coefficient, assuming that kinetic effects appear only in the amplitude and not in the polarization of the wave (see, e.g., [17] and [18]). For AUG, in Fig. 3 the spatial profiles of $E, j$ are presented. The absorption of the wave occurs in the narrow resonance layer near the plasma center. The damping profile is very steep, indicating large values of the conductivity tensor within the resonance layer. The current density increases slowly towards the resonance layer, becomes much larger due to the resonant interaction and then it is nullified past the resonance, since the electric field of the wave has been completely damped.

In Fig. 4 we give the profiles of $E$ and $j$ for parameters relevant to ITER. In this case, due to the specific value of the magnetic field on the axis, the resonance layer is shifted towards the high-field side. From Fig. 4(b) it is seen that the current in the plasma is very localized, practically only inside the resonance layer. Contrary to the hot plasma X-mode [see Fig. 3(b)], the polarization of the O-mode is such that current cannot be generated before the wave reaches the resonance.

As an outcome, a benchmarking of our method is essential. This can be done in terms of asymptotic techniques, the results of which can be directly compared to our numerical results. For the specific benchmark we utilize the beam tracing method. The numerical implementation is done by the code NGBT, which solves the beam tracing equations in plane geometry for arbitrary beam shape [19]. Unlike ECFW, which models the plasma response in terms of the fully kinetic tensor, NGBT follows the mainstream of codes based on asymptotic methods and considers cold plasma propagation, with kinetic effects reflected only in the reduction of the amplitude according to the hot plasma optical depth.

In Fig. 5 the results of ECFW are compared to NGBT for the same parameters as the ones in Fig. 3. One can see that, despite the difference in the physics modelling, the agreement between the results of the two codes is very good. This happens because, in the plain slab model, absorption is described entirely by the anti-Hermitian part of the dielectric tensor. Consequently, 

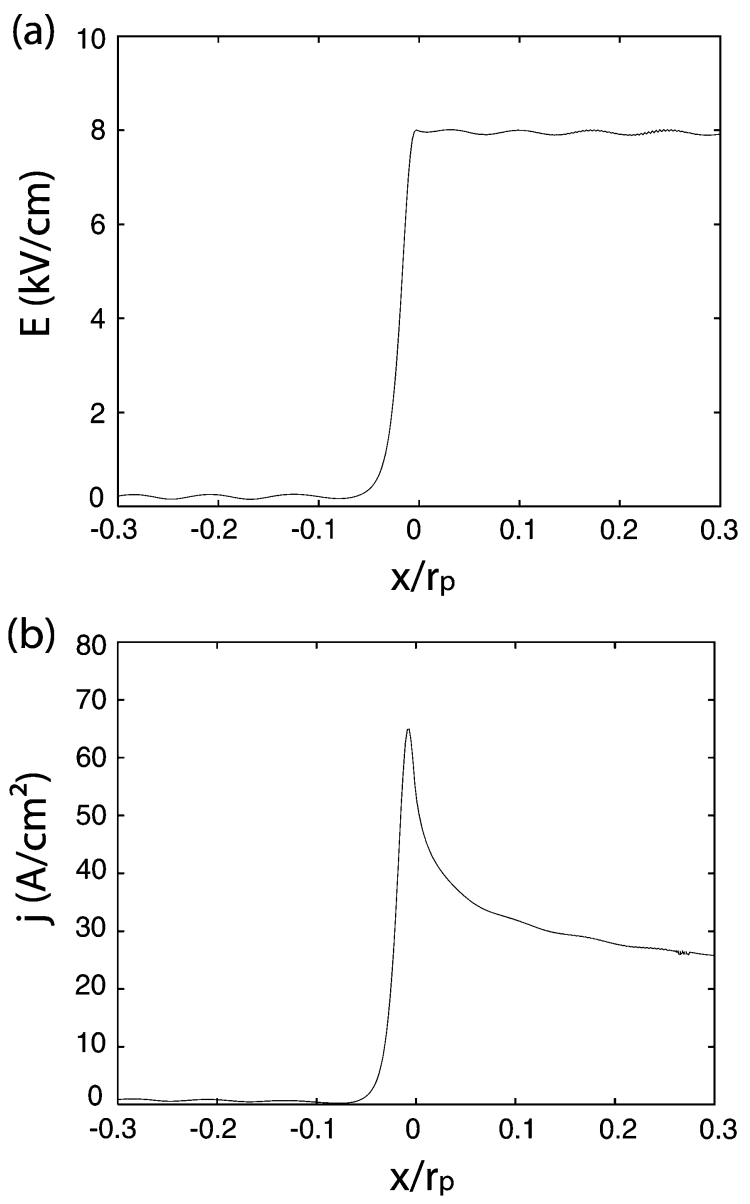

Fig. 3. FDTD simulation of hot, weakly-relativistic plasma for parameters relevant to ECRH in AUG: Steady-state spatial profiles of (a) $E$ and (b) $j$.

the power deposition as a function of $x$ becomes completely independent of details of the beam trajectory. In particular, the optical depth of the plasma slab is not changed by the effects of kinetic dispersion on beam propagation (see [7] for details).

\section{CONCLUSION-DISCUSSION}

We present a full-wave model of EC propagation in hot anisotropic plasma, based on the FDTD method. For this type of problem, frequency-domain asymptotic methods are most popular, because of the ability to derive analytic expressions, in some cases even exact, for the plasma response. For a constant-frequency wave propagating in stationary plasma (the most common approach to ECRH), FDTD can be applied directly using the dielectric response of the plasma as derived in the frequency domain. The scattered-field formulation for anisotropic plasma is derived in compact form, as a continuation of [14]. A numerical application is presented in slab geometry, allowing a simpler description of the plasma-wave coupling, based on the 1D implementation of FDTD. The code ECFW studies the phenomena under different physics models for the dielectric plasma response.

The main numerical results are summarized as follows: In all cases, the fields reach a steady-state after a characteristic time, as expected due to the lack of time dependence in the plasma
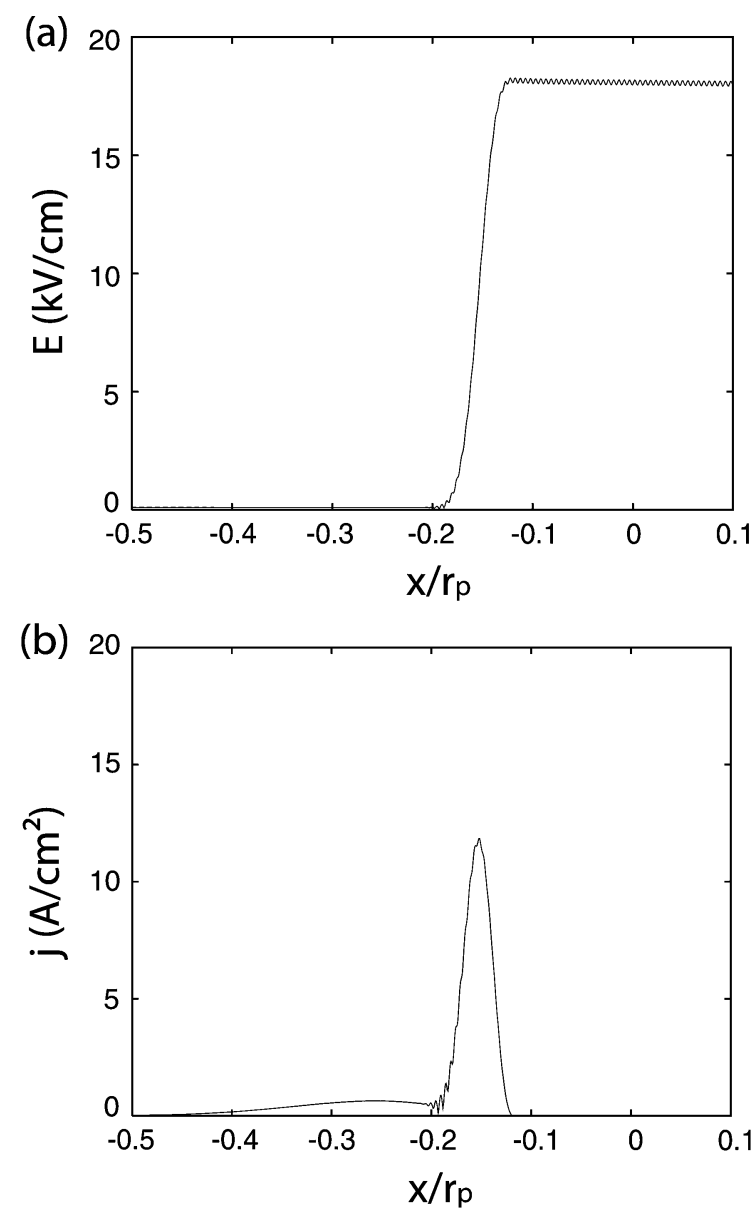

Fig. 4. FDTD simulation of hot weakly-relativistic plasma for ITER parameters: Steady-state spatial profiles of (a) $E$ and (b) $j$.

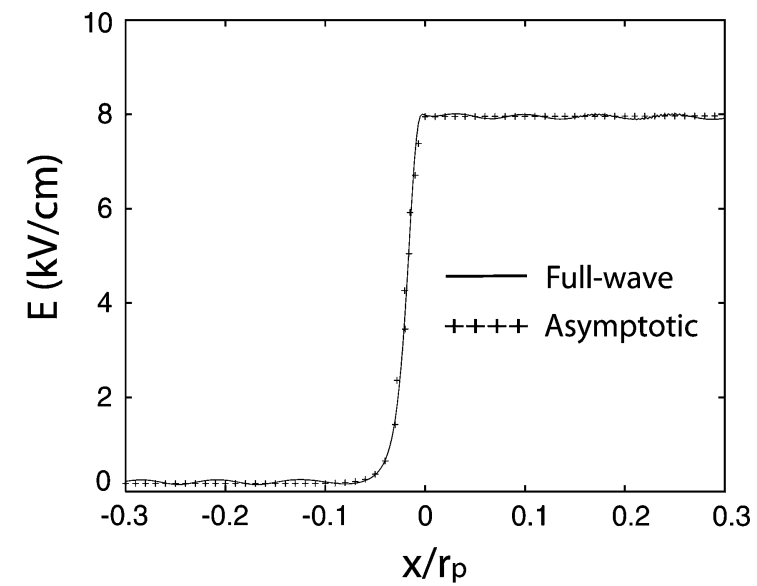

Fig. 5. Comparison of the full-wave solution (ECFW) and the beam-tracing asymptotic solution (NGBT) for AUG parameters.

response. For cold/warm plasma, the electric field amplitude remains constant along propagation, because these models do not include the effect of cyclotron absorption. In hot plasma, the absorption of the wave is found to occur in a very narrow region, known as the resonance layer, which is defined by the wave frequency and the magnetic field profile. In specific, the position of the resonance layer on the $x$-axis is controllable, i.e., it can be shifted towards the low- or the high-field side, by changing 
either the wave frequency or the magnetic field on the axis. The generated current is also very localized, practically only inside the resonance layer. Benchmarking of ECFW with a code based on the beam tracing asymptotic method proved successful.

A discussion on the limitations of our model is necessary. First, in the 1D description it is not possible to model 2D propagation and associated effects, like, e.g., mode conversion, ECCD, as well as effects having to do with the variation of the electric field profile (focusing, diffraction). These issues can be studied only under a 2D/3D implementation of FDTD, which is the subject of future work. Another limitation is that the dielectric response of the plasma considered here is in terms of linear theory. Hence, the description of nonlinear wave-particle interaction is missing. In this direction, an immediate extension of our work is to calculate the current density based on particles moving on the grid. The particle orbits may be calculated by following the discretized equations of motion or by other available methods.

\section{ACKNOWLEDGMENT}

C. Tsironis would like to thank Drs. E. Poli, R. Bilato, and G. V. Pereverzev for the useful discussions.

\section{REFERENCES}

[1] R. Prater, "Heating and current drive by electron-cyclotron waves," Phys. Plasmas, vol. 11, pp. 2349-2376, 2004.

[2] L. Friedland and I. B. Bernstein, "Geometric optics in plasmas characterized by non-Hermitian dielectric tensors," Phys. Rev. A, vol. 22, pp. $1680-1685,1980$.

[3] A. G. Peeters, "Extension of the ray equations of geometric optics to included diffraction effects," Phys. Plasmas, vol. 3, pp. 4386-4395, 1996.

[4] G. V. Pereverzev, "Beam tracing in inhomogeneous anisotropic plasma," Phys. Plasmas, vol. 5, pp. 3529-3541, 1998.

[5] D. G. Swanson, Plasma Waves. Bristol, U.K.: IoP Press, 2003.

[6] J. L. Segui, Y. Michelot, D. Vezard, L. Laurent, and G. Giruzzi, "An overview of ECA results in Tore Supra," in Proc. 9th Joint Workshop on ECE and ECH, Borrego Springs, CA, 1995, pp. 223-228.

[7] E. Westerhof, "Wave propagation through an electron cyclotron resonance layer," Plasma Phys. Control. Fusion, vol. 39, pp. 1015-1029, 1997.

[8] H. Muta, Y. Ueda, and Y. Kawai, "One dimensional simulation of microwave propagation in electron cyclotron resonance plasmas," Jpn. J. Appl. Phys., vol. 36, pp. 872-876, 1997.

[9] H. Muta, T. Sakoda, Y. Ueda, and Y. Kawai, "Three dimensional simulation of microwave propagation in an electron cyclotron resonance plasma," Jpn. J. Appl. Phys., vol. 36, pp. 4773-4776, 1997.

[10] F. D. Silva, "Simulation of reflectometry density changes using a 2D full-wave code," Rev. Sci. Instrum., vol. 72, pp. 311-314, 2001.

[11] H. Hojo, A. Fukuchi, and A. Itakura, "Full-wave simulations on ultrashort-pulse reflectometry for helical plasmas," Rev. Sci. Instrum., vol. 75 , pp. $3813-3815,2004$

[12] K. S. Yee, "Numerical solution of initial boundary value problems involving Maxwells equations in isotropic media," IEEE Tram. Antennas Propag., vol. 14, pp. 302-307, 1966.

[13] G. Mur, "Absorbing boundary conditions for finite-difference approximation of the time-domain electromagnetic-field equations," IEEE Trans. Electromagn. Compat., vol. 23, pp. 1073-1078, 1981.

[14] J. Schneider and S. Hudson, "The finite-difference time-domain method applied to anisotropic material," IEEE Trans. Antennas Propag., vol. 41, p. 994, 1993.
[15] P. A. Robinson, "Relativistic plasma dispersion functions," J. Math. Phys., vol. 27, pp. 1206-1214, 1986.

[16] K. S. Kunz and R. J. Luebbers, The Finite Difference Time Domain Method for Electromagnetics. Boca Raton, FL: CRC Press, 1992.

[17] E. Westerhof, "Implementation of TORAY at JET," FOM Rijnhuizen, Nieugewein, The Netherlands, Tech. Rep. RR 89-183, 1989.

[18] E. Poli, A. G. Peeters, and G. V. Pereverzev, "TORBEAM, a beam tracing code for electron-cyclotron waves in tokamak plasmas," Comput. Phys. Commun., vol. 136, pp. 90-104, 2001.

[19] C. Tsironis, E. Poli, and G. V. Pereverzev, "Beam tracing description of non-Gaussian wave beams," Phys. Plasmas, vol. 13, pp. 113304-113313, 2006.

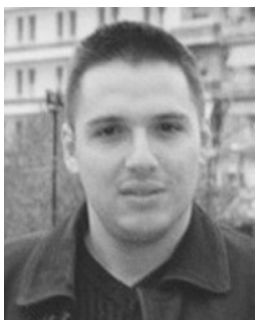

Christos Tsironis received the Physics degree and the M.Sc. degree in physics from the National and Kapodistrian University of Athens, Athens, Greece, in 1999 an 2002, respectively, and the Ph.D. degree from Aristotle University of Thessaloniki, Thessaloniki, Greece, in 2007.

From 1999 to 2000, he was with the Nonlinear Dynamical Systems Analysis Group, National and Kapodistrian University of Athens. In 2003, he joined the Plasma Astrophysics Group, Aristotle University of Thessaloniki, where he is currently a Postdoctoral Associate. He has been a Research Associate of the Association EURATOM-Hellenic Republic since 1999. His research interests include nonlinear dynamics, wave physics and plasma physics with applications in astrophysics and thermonuclear fusion.

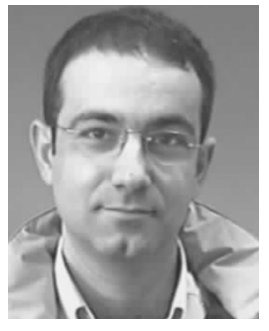

Theodoros Samaras (S'93-A'97-M'02) received the physics degree from Aristotle University of Thessaloniki, Thessaloniki, Greece, in 1990, the M.Sc. degree in medical physics (with distinction) from the University of Surrey, Surrey, U.K., in 1991, and the Ph.D. degree from the Aristotle University of Thessaloniki, Thessaloniki, Greece, in 1996.

In 1998, he was with the Bioelectromagnetics/Electromagnetic Compatibility Group, Swiss Federal Institute of Technology, where he was involved with studying the temperature increase due to the absorption of electromagnetic energy in dispersive biological materials and the effect of heat diffusion in electromagnetic dosimetry. He subsequently joined the Hyperthermia Unit, Erasmus Medical Center, Rotterdam, The Netherlands, where he conducted research on treatment quality of superficial hyperthermia. In December 1999, he returned to the Aristotle University of Thessaloniki, where he is currently an Assistant Professor. His research interests include computational electromagnetics and numerical techniques with applications in biomedical technology, EMC, and telecommunications.

Dr. Samaras was the recipient of a Marie Curie Fellowship awarded by the European Commission.

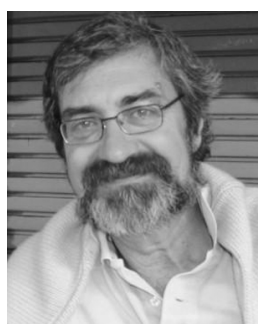

Loukas Vlahos received the physics degree from Aristotle University of Thessaloniki, Thessaloniki, Greece, in 1971, the M.Sc. degree in physics from the University of Maryland, Maryland, U.S.A., in 1976, and the Ph.D. degree from the University of Maryland, Baltimore, in 1979.

After completing his Ph.D., he worked for approximately five years at the University of Maryland as a Senior Research Associate. In 1985, he joined the faculty of the Aristotle University of Thessaloniki, where he is currently a Professor. Since 1990, he has developed a research group, funded mainly by the European Union and the Greek government, that works on problems of high energy astrophysics, plasma physics, turbulence and nonlinear dynamics. 